\title{
Approximation of Parametric Derivatives by the Empirical Interpolation Method*
}

\author{
Jens L. Eftang ${ }^{\dagger}$, Martin A. Grepl ${ }^{\ddagger}$, \\ Anthony T. Patera ${ }^{\S}$ and Einar M. Rønquist $\llbracket$
}

Bericht Nr. 330

September 2011

Key words: function approximation; a priori convergence;

Empirical Interpolation Method; parametric derivatives; sensitivity derivatives

AMS (MSC 2010) subject classifications: G5G99, 65D05, 41A05, 41A25

Institut für Geometrie und Praktische Mathematik RWTH Aachen

Templergraben 55, D-52056 Aachen (Germany)

* Submitted to Foundations of Computational Mathematics (Springer), September 26th, 2011

$\dagger$

Department of Mechanical Engineering, Massachusetts Institute of Technology, 77 Massachusetts Avenue, Cambridge, MA 02139, USA; corresponding author, eftang@mit.edu

† RWTH Aachen University, Numerical Mathematics, Templergraben 55, 52056 Aachen, Germany

$\S$

Department of Mechanical Engineering, Massachusetts Institute of Technology, 77 Massachusetts Avenue, Cambridge, MA 02139, USA

ॠ Department of Mathematical Sciences, Norwegian University of Science and Technology, NO-7491 Trondheim, Norway 


\title{
Approximation of Parametric Derivatives by the Empirical Interpolation Method*
}

\author{
Jens L. Eftang ${ }^{\dagger} \quad$ Martin A. Grepl ${ }^{\ddagger}$ Anthony T. Patera ${ }^{\S}$ \\ Einar M. Rønquist
}

September 26, 2011

\begin{abstract}
We introduce a general a priori convergence result for the approximation of parametric derivatives of parametrized functions. We show that for a given approximation scheme the approximations of parametric derivatives of a given parametrized function are convergent provided that the approximation of the function itself is convergent. The assumptions on the approximation scheme are rather weak; for example we may consider both projection-based and interpolation-based approximation. We present numerical results with one particular interpolation scheme - the Empirical Interpolation Method - to confirm the validity of the general theory.
\end{abstract}

Keywords: function approximation; a priori convergence; Empirical Interpolation Method; parametric derivatives; sensitivity derivatives

AMS (MSC 2010) subject classification: G5G99, 65D05, 41A05, 41A25

\section{Introduction}

In contexts such as product design, shape optimization, and parameter estimation it is crucial to understand the behavior of a given physical system as a function of parameters that describe the system in terms of for example materials, shapes, or operation conditions. Typically, the goal is to minimize a

\footnotetext{
*Submitted to Foundations of Computational Mathematics (Springer), September 26th, 2011

${ }^{\dagger}$ Department of Mechanical Engineering, Massachusetts Institute of Technology, 77 Massachusetts Avenue, Cambridge, MA 02139, USA; corresponding author, eftang@mit.edu

${ }^{\ddagger}$ RWTH Aachen University, Numerical Mathematics, Templergraben 55, 52056 Aachen, Germany

$\S$ Department of Mechanical Engineering, Massachusetts Institute of Technology, 77 Massachusetts Avenue, Cambridge, MA 02139, USA

IDepartment of Mathematical Sciences, Norwegian University of Science and Technology, NO-7491 Trondheim, Norway
} 
parameter dependent cost functional related to certain quantities derived from the state of the system. To this end an automatic optimization algorithm may be employed. Such algorithms typically necessitates calculation of derivatives of the cost functional with respect to the parameters (parametric or sensitivity derivatives). This calculation may be performed directly on the cost functional (by finite differences), or through parametric derivatives of the state [3].

The state of a parametrized physical system can typically be viewed as a parameter dependent function implicitly defined as the solution of a parametrized partial or ordinary differential equation. In this paper, we develop a new $a$ priori convergence theory that for a given approximation scheme demonstrates that the approximations of parametric derivatives of a given parametrized function are convergent provided that the approximation of the function itself is convergent. The assumptions on the approximation scheme are rather weak. We restrict here attention to parameter dependent functions that are defined explicitly, i.e., functions that in addition to spatial variables have an explicit dependence on one or several scalar parameters. However in principle our new theory also applies to cases in which the parametrized function is defined implicitly as the solution to a differential equation.

The Empirical Interpolation Method (EIM), introduced in [1, 8], is an interpolation method developed specifically for the approximation of parametrized functions. ${ }^{1}$ The new convergence theory presented in this paper is developed with the EIM in mind, and is discussed and applied within the context of the EIM. However, our theoretical results also apply to rather general approximation schemes other than the EIM; in particular, we may consider both projectionbased and interpolation-based approximation. The main limitations of the theory are related to regularity assumptions in space and parameter on the parametrized function, and on the particular norms that may be considered.

The results in this paper have several useful implications. First, if the EIM is employed for evaluation of an objective function subject to optimization typically an integral - with respect to a set of parameters, our theory suggests that we may accurately compute the parametric Jacobian and Hessian matrices without expensive generation of additional EIM spaces. Second, the rigorous $a$ posteriori bounds for the error in the EIM approximation recently introduced in [6] depend on the error in the EIM approximation of parametric derivatives at a finite number of points in the parameter domain; smaller errors for these EIM derivative approximations imply sharper EIM error bounds.

The remainder of the paper is organized as follows. First, in Section 2 we introduce necessary notation and recall some results from polynomial approximation theory. Next, in Section 3, we present the new general a priori convergence result. Then, in Section 4 we review the EIM and apply the new

\footnotetext{
${ }^{1}$ In particular, the EIM serves to construct parametrically affine approximations of parameter dependent non-affine or non-linear differential operators within the Reduced Basis (RB) framework for parametric reduced order modelling of partial differential equations [13]. An affine representation (or approximation) of the operator allows an efficient "offline-online" computational decoupling, which in turn is a crucial ingredient in the RB computational framework. We refer to $[7,8]$ for the application of the EIM for RB approximations.
} 
convergence theory in this particular context. Subsequently, in Section 5, we validate the theory through two numerical examples for which the functions to be approximated have different parametric smoothness. Finally, in Section 6, we provide some concluding remarks.

\section{Preliminaries}

\subsection{Notation}

We denote by $\Omega \subset \mathbb{R}^{d}$ the spatial domain $(d=1,2,3)$; a particular point $x \in \Omega$ shall be denoted by $x=\left(x_{(1)}, \ldots, x_{(d)}\right)$. We denote by $\mathcal{D}=[-1,1]^{P} \subset \mathbb{R}^{P}$ the parameter domain $(P \geq 1)$; a particular parameter value $\mu \in \mathcal{D}$ shall be denoted by $\mu=\left(\mu_{(1)}, \ldots, \mu_{(P)}\right)$. We choose $\mathcal{D}=[-1,1]^{P}$ for the sake of simplicity in our theoretical arguments; our results remain valid for any parameter domain that maps to $[-1,1]^{P}$ through an affine transformation.

We introduce a parametrized function $\mathcal{F}: \Omega \times \mathcal{D} \rightarrow \mathbb{R}$ for which we assume $\mathcal{F}(\cdot ; \mu) \in L^{\infty}(\Omega)$ for all $\mu \in \mathcal{D}$; here, $L^{\infty}(\Omega)=\left\{v: \operatorname{ess}_{\sup } \in \Omega|v(x)|<\infty\right\}$. We then introduce a multi-index of dimension $P$,

$$
\beta=\left(\beta_{1}, \ldots, \beta_{P}\right),
$$

where the entries $\beta_{i}, 1 \leq i \leq P$, are non-negative integers. We define for any multi-index $\beta$ the parametric derivatives of $\mathcal{F}$,

$$
\mathcal{F}^{(\beta)}=\frac{\partial^{|\beta|} \mathcal{F}}{\partial \mu_{(1)}^{\beta_{1}} \cdots \partial \mu_{(P)}^{\beta_{P}}},
$$

where

$$
|\beta|=\sum_{i=1}^{P} \beta_{i}
$$

is the length of $\beta$ and hence the differential order. Given the parameter domain dimension $P$, we denote the set of all distinct multi-indices $\beta$ of length $p$ by $\mathcal{M}_{p}$.

For purposes of our theoretical arguments later we assume, for all $x \in \Omega$, that $\mathcal{F}(x ; \cdot) \in C^{1}(\mathcal{D})$ and that $\sup _{\mu \in \mathcal{D}}\left|\mathcal{F}^{(\beta)}(x ; \mu)\right|<\infty$ for all $\beta \in \mathcal{M}_{2}$. Here $C^{s}(\mathcal{D})$ denotes the space of functions with continuous $s$-order parametric derivatives over $\mathcal{D}$.

Also for purposes of our theoretical arguments we shall write $\mathcal{D}$ as the tensor product $\mathcal{D}=\mathcal{D}_{(1)} \times \cdots \times \mathcal{D}_{(P)}$, where $\mathcal{D}_{(i)}=[-1,1], 1 \leq i \leq P$. We shall further consider any particular parameter dimension $\mathcal{S} \equiv \mathcal{D}_{j}, 1 \leq j \leq P$. In this case we fix the $P-1$ parameter values $\mu_{(i)} \in \mathcal{D}_{(i)}, 1 \leq i \leq P, i \neq j$, and we introduce for simplicity of notation the function $\mathcal{J}_{\beta, j}: \Omega \times \mathcal{S} \rightarrow \mathbb{R}$ defined for $x \in \Omega$ and $y \in \mathcal{S}$ by

$$
\mathcal{J}_{\beta, j}(x ; y) \equiv \mathcal{F}^{(\beta)}\left(x ;\left(\mu_{(1)}, \ldots, \mu_{(j-1)}, y, \mu_{(j+1)}, \ldots, \mu_{(P)}\right)\right) .
$$




\subsection{Polynomial Interpolation}

In this section we recall some results from polynomial interpolation theory. We first describe a general interpolation framework for which we state three hypotheses. These hypotheses are key ingredients in the proof of our new convergence theory in Section 3.

Let $\Gamma=[-1,1]$, and let $\mathcal{H}$ denote a family of functions such that each $h \in \mathcal{H}$ is a function $\Gamma \rightarrow \mathbb{R}$ wich sufficient regularity. We introduce $N+1$ distinct interpolation nodes $y_{N, i} \in \Gamma, 0 \leq i \leq N$, and $N+1$ characteristic functions $\chi_{N, i}, 0 \leq i \leq N$, that satisfy $\chi_{N, i}\left(y_{N, j}\right)=\delta_{i, j}, 0 \leq i, j \leq N$; here, $\delta_{i, j}$ is the Kronecker delta symbol. We finally introduce an interpolation operator $I_{N}$ defined for any function $h \in \mathcal{H}$ by $I_{N} h=\sum_{i=0}^{N} h\left(y_{N, i}\right) \chi_{N, i}$. We may now formally state our three hypotheses.

Hypothesis 1. For all $h \in \mathcal{H}$ the error in the derivative of the interpolant $I_{N} h$ satisfies

$$
\left|h^{\prime}(y)-\left(I_{N} h\right)^{\prime}(y)\right| \leq G(N), \quad \forall y \in \Gamma,
$$

where the function $G: \mathbb{N} \rightarrow(0, \infty)$ is independent of $h$ and satisfies $G(N) \rightarrow 0$ as $N \rightarrow \infty$.

Hypothesis 2. The characteristic functions $\chi_{N, i}, 0 \leq i \leq N$, satisfy

$$
\sum_{i=0}^{N}\left|\chi_{N, i}^{\prime}(y)\right| \leq D(N), \quad \forall y \in \Gamma,
$$

where the function $D: \mathbb{N} \rightarrow(0, \infty)$ satisfies $D(N) \rightarrow \infty$ as $N \rightarrow \infty$.

Hypothesis 3. Let $\epsilon \in \mathbb{R}^{+}$. As $\epsilon \rightarrow 0$ the solution $N_{\text {bal }}=N_{\text {bal }}(\epsilon)>0$ to the equation

$$
G\left(N_{\mathrm{bal}}\right)=D\left(N_{\mathrm{bal}}\right) \epsilon
$$

satisfies

$$
H(\epsilon) \equiv \epsilon D\left(N_{\text {bal }}(\epsilon)\right) \rightarrow 0 .
$$

We next consider several interpolation schemes and in each case confirm the corresponding instantiations of our hypotheses under suitable regularity conditions on the functions in the family $\mathcal{H}$. First, we assume for all $h \in \mathcal{H}$ that $h \in C^{1}(\Gamma)$ and furthermore that $\sup _{y \in \Gamma}\left|h^{\prime \prime}(y)\right|<\infty$; we then consider piecewise linear interpolation over equidistant interpolation nodes $y_{N, i}=(2 i / N-1) \in \Gamma$, $0 \leq i \leq N$. In this case the characteristic functions $\chi_{N, i}$ are continuous and piecewise linear "hat functions" with support only on the interval $\left[y_{N, 0}, y_{N, 1}\right]$ for $i=0$, only on the interval $\left[y_{N, i-1}, y_{N, i+1}\right]$ for $1 \leq i \leq N-1$, and only on the interval $\left[y_{N, N-1}, y_{N, N}\right]$ for $i=N$. For piecewise linear interpolation Hypothesis 1 and Hypothesis 2 obtain for

$$
\begin{aligned}
& G(N)=2 N^{-1} \sup _{h \in \mathcal{H}}\left\|h^{\prime \prime}\right\|_{L^{\infty}(\Gamma)} \equiv c_{\mathcal{H}}^{\operatorname{lin}} N^{-1}, \\
& D(N)=N,
\end{aligned}
$$


respectively, where $c_{\mathcal{H}}^{\operatorname{lin}}=2 \sup _{h \in \mathcal{H}}\|h\|_{L^{\infty}(\Gamma)}$. In this case (6) in Hypothesis 2 obtains with equality. We include the proofs in Appendix A.1. It is straightforward to demonstrate Hypothesis 3: we note that in this case (7) has the solution

$$
N_{\text {bal }}(\epsilon)=\left(2 \sup _{h \in \mathcal{H}} \frac{\left\|h^{\prime \prime}\right\|_{L^{\infty}(\Gamma)}}{\epsilon}\right)^{1 / 2}=\left(\frac{c_{\mathcal{H}}^{\operatorname{lin}}}{\epsilon}\right)^{1 / 2}
$$

and hence that

$$
H(\epsilon)=\left(c_{\mathcal{H}}^{\operatorname{lin}} \epsilon\right)^{1 / 2} \rightarrow 0
$$

as $\epsilon \rightarrow 0$.

Next, we assume for all $h \in \mathcal{H}$ that $h \in C^{2}(\Gamma)$ and furthermore that $\sup _{y \in \Gamma}\left|h^{\prime \prime \prime}(y)\right|<\infty$; we then consider piecewise quadratic interpolation over equidistant interpolation nodes $y_{N, i}=(2 i / N-1) \in \Gamma, 0 \leq i \leq N$. We assume that $N$ is even such that we may divide $\Gamma$ into $N / 2$ intervals $\left[y_{N, i}, y_{N, i+2}\right]$, for $i=0,2,4, \ldots, N-2$. The characteristic functions are for $y \in\left[y_{N, i}, y_{N, i+2}\right]$ then given as

$$
\begin{aligned}
\chi_{N, i}(y) & =\frac{\left(y-y_{N, i+1}\right)\left(y-y_{N, i+2}\right)}{2 h^{2}}, \\
\chi_{N, i+1}(y) & =\frac{\left(y-y_{N, i}\right)\left(y-y_{N, i+2}\right)}{-h^{2}}, \\
\chi_{N, i+2}(y) & =\frac{\left(y-y_{N, i}\right)\left(y-y_{N, i+1}\right)}{2 h^{2}},
\end{aligned}
$$

for $i=0,2,4, \ldots, N-2$, where $h=2 / N=y_{N, j+1}-y_{N, j}, 0 \leq j \leq N-1$. For piecewise quadratic interpolation Hypothesis 1 and Hypothesis 2 obtain for

$$
\begin{aligned}
G(N) & =28 N^{-2} \sup _{h \in \mathcal{H}}\left\|h^{\prime \prime \prime}\right\|_{L^{\infty}(\Gamma)} \equiv c_{\mathcal{H}}^{\text {quad }} N^{-2}, \\
D(N) & =\frac{5}{2} N
\end{aligned}
$$

respectively, where $c_{\mathcal{H}}^{\text {quad }}=28 \sup _{h \in \mathcal{H}}\left\|h^{\prime \prime \prime}\right\|_{L^{\infty}(\Gamma)}$. We include the proofs in Appendix A.2. It is straightforward to demonstrate Hypothesis 3: we note that in this case (7) has the solution

$$
N_{\text {bal }}(\epsilon)=\left(\frac{56 \sup _{h \in \mathcal{H}}\left\|h^{\prime \prime \prime}\right\|_{L^{\infty}(\Gamma)}}{5 \epsilon}\right)^{1 / 3}=\left(\frac{2 c_{\mathcal{H}}^{\text {quad }}}{5 \epsilon}\right)^{1 / 3}
$$

and hence that

$$
H(\epsilon)=\frac{5}{2}\left(\frac{2}{5} c_{\mathcal{H}}^{\text {quad }}\right)^{1 / 3} \epsilon^{2 / 3} \rightarrow 0
$$

as $\epsilon \rightarrow 0$. 
Finally, we assume for all $h \in \mathcal{H}$ that $h$ is analytic over $\Gamma$ and consider standard Chebyshev interpolation over the Chebyshev nodes $y_{N, i}=-\cos (i \pi / N)$, $0 \leq i \leq N$. The characteristic functions are in this case the Lagrange polynomials $\chi_{N, i} \in \mathbb{P}_{N}(\Gamma)$ that satisfy $\chi_{N, i}\left(y_{N, j}\right)=\delta_{i, j}, 0 \leq i, j \leq N$. For Chebyshev interpolation Hypothesis 1 and Hypothesis 2 obtain for

$$
\begin{aligned}
G(N) & =c_{\mathcal{H}}^{\mathrm{Cheb}} N e^{-N \log \left(\rho_{\mathcal{H}}\right)}, \quad(N>0) \\
D(N) & =N^{2},
\end{aligned}
$$

respectively, where $c_{\mathcal{H}}^{\text {Cheb }}>0$ and $\rho_{\mathcal{H}}>1$ depend only on $\mathcal{H}$.

A proof of $(20)$ (realized for an analytic function $h \in \mathcal{H}$ ) can be found in [11]. A similar but somewhat less optimal result is obtained in [14, Eq. (4.18)]. However the result in [14] holds only for the maximum error over the $N+1$ Chebyshev interpolation nodes. In [11] this result is improved and extended to a bound for the maximum pointwise error over the entire interval $[-1,1]$. We note that results similar to (21) (arbitrary high algebraic order convergence for smooth functions) are common in the literature for $L^{2}$ or Sobolev norms; see for example $[2,4,14]$. The pointwise exponential estimate required for our theoretical derivation in this paper proved more difficult to find.

The result (6) in Hypothesis 2 obtains in this case with equality. We refer to [12, pp. 119-121] for a proof.

We finally demonstrate Hypothesis 3: we let $c=c_{\mathcal{H}}^{\text {Cheb }}, \eta=\eta_{\mathcal{H}}=\log \left(\rho_{\mathcal{H}}\right)>$ 0 , and we note that in this case (7) yields the transcendental equation

$$
c N e^{-N \eta}=N^{2} \epsilon \text {. }
$$

which admits the solution

$$
N_{\text {bal }}(\epsilon)=\frac{1}{\eta} \mathcal{W}\left(\frac{c \eta}{\epsilon}\right)
$$

where $\mathcal{W}$ denotes the Lambert $W$ function(s) [5] defined by $\xi=\mathcal{W}(\xi) e^{\mathcal{W}(\xi)}$ for any $\xi \in \mathbb{C}$.

We now note that for real $\xi>e$, we have $\mathcal{W}(\xi)<\log (\xi)$. We demonstrate the proof in Appendix A.3. Thus, for sufficiently large $\epsilon$ such that $c \eta / \epsilon>e$, we obtain

$$
N_{\text {bal }}(\epsilon)<\frac{1}{\eta} \log \left(\frac{c \eta}{\epsilon}\right)=\frac{1}{\eta}(\log (c \eta)+\log (1 / \epsilon)) \leq A \log (1 / \epsilon)
$$

for some sufficiently large constant $A$. Hence in this case

$$
H(\epsilon)<\epsilon A^{2}(\log (1 / \epsilon))^{2}
$$

for $\epsilon$ sufficiently small. We now consider $H(\epsilon)$ as $\epsilon \rightarrow 0$. By application of 
L'Hôpital's rule twice (Eqs. (28) and (30) below) we obtain

$$
\begin{aligned}
\lim _{\epsilon \rightarrow 0} H(\epsilon) & <A^{2} \lim _{\epsilon \rightarrow 0} \epsilon(\log (1 / \epsilon))^{2} \\
& =A^{2} \lim _{\epsilon \rightarrow 0} \frac{(\log (\epsilon))^{2}}{1 / \epsilon} \\
& =A^{2} \lim _{\epsilon \rightarrow 0} \frac{2 \log (\epsilon) / \epsilon}{-1 / \epsilon^{2}} \\
& =2 A^{2} \lim _{\epsilon \rightarrow 0} \frac{\log (\epsilon)}{-1 / \epsilon} \\
& =2 A^{2} \lim _{\epsilon \rightarrow 0} \frac{1 / \epsilon}{1 / \epsilon^{2}} \\
& =2 A^{2} \lim _{\epsilon \rightarrow 0} \epsilon=0 .
\end{aligned}
$$

Hypothesis 3 thus holds.

\section{A General A Priori Convergence Result}

We introduce an approximation space $W_{M} \equiv W_{M}(\Omega)$ of finite dimension $M$. For any $\mu \in \mathcal{D}$, our approximation to the function $\mathcal{F}(\cdot ; \mu): \Omega \rightarrow \mathbb{R}$ shall reside in $W_{M}$; the particular approximation procedure invoked is not relevant for our theoretical results in this section. We show here that if, for any $\mu \in \mathcal{D}$, the error in the best $L^{\infty}(\Omega)$ approximation to $\mathcal{F}(\cdot ; \mu)$ in $W_{M}$ goes to zero as $M \rightarrow \infty$, then, for any multi-index $\beta,|\beta| \geq 0$, the error in the best $L^{\infty}(\Omega)$ approximation to $\mathcal{F}^{(\beta)}(\cdot ; \mu)$ in $W_{M}$ also goes to zero as $M \rightarrow \infty$. Of course, only modest $M$ are of interest in practice: the computational cost associated with the approximation is $M$-dependent. However, our theoretical results in this section provide some promise that we may in practice invoke the "original" approximation space and approximation procedure also for the approximation of parametric derivatives.

We introduce, for any fixed $p \geq 0$ and any $M \geq 1$,

$$
e_{M}^{p} \equiv \max _{\beta \in \mathcal{M}_{p}} \max _{\mu \in \mathcal{D}} \inf _{w \in W_{M}}\left\|\mathcal{F}^{(\beta)}(\cdot ; \mu)-w\right\|_{L^{\infty}(\Omega)} .
$$

We then recall the definition of $\mathcal{J}_{\beta, j}$ from (4), and state

Proposition 1. Let $p$ be a fixed non-negative integer. Assume that Hypotheses 1, 2, and 3 hold for the family of functions $\mathcal{H}$ given by

$$
\mathcal{H}=\left\{\mathcal{J}_{\beta, j}(x ; \cdot): x \in \Omega, \beta \in \mathcal{M}_{p}, 1 \leq j \leq P\right\} .
$$

In this case, if $e_{M}^{p} \rightarrow 0$ as $M \rightarrow \infty$, then

$$
e_{M}^{p+1} \rightarrow 0
$$

as $M \rightarrow \infty$. 
Proof. For each $x \in \Omega$, and for given $\beta \in \mathcal{M}_{p}$ and $j, 1 \leq j \leq P$, we first introduce the interpolant $\mathcal{J}_{N, \beta, j}(x ; \cdot) \equiv I_{N} \mathcal{J}_{\beta, j}(x ; \cdot) \in \mathbb{P}_{N}(\mathcal{S})$ given by

$$
\mathcal{J}_{N, \beta, j}(x ; \cdot) \equiv I_{N} \mathcal{J}_{\beta, j}(x ; \cdot)=\sum_{i=0}^{N} \mathcal{J}_{\beta, j}\left(x ; y_{N, i}\right) \chi_{N, i}(\cdot) ;
$$

recall that here, $\mathcal{S}=\mathcal{D}_{(j)}=[-1,1]$, and $\chi_{N, i}: \mathcal{S} \rightarrow \mathbb{R}, 0 \leq i \leq N$, are characteristic functions that satisfy $\chi_{N, i}\left(y_{N, j}\right)=\delta_{i, j}, 0 \leq i, j \leq N$.

We then introduce functions

$$
\begin{aligned}
w_{\beta, j}^{*}\left(\cdot ; \mu_{(1)}, \ldots, \mu_{(j-1)}, y, \mu_{(j+1)}, \ldots, \mu_{(P)}\right) & \equiv w_{\beta, j}^{*}(\cdot ; y) \\
& \equiv \arg \inf _{w \in W_{M}}\left\|\mathcal{J}_{\beta, j}(\cdot ; y)-w\right\|_{L^{\infty}(\Omega)}
\end{aligned}
$$

for any $y \in \mathcal{S} .^{2}$ Next, for all $(x, y) \in \Omega \times \mathcal{S}$, we consider an approximation to $\mathcal{J}_{\beta, j}(x ; y)$ given by $\sum_{i=0}^{N} \chi_{N, i}(y) w_{\beta, j}^{*}\left(x ; y_{N, i}\right)$. Note that this approximation is just an interpolation between the optimal approximations at the interpolation nodes $y_{N, i} \in \mathcal{S}$ and hence that for $y=y_{N, i}$ this approximation is exact. We next let ' denote differentiation with respect to the variable $y$ and consider the error in the derivative of this approximation. By the triangle inequality we obtain

$$
\begin{aligned}
& \left\|\mathcal{J}_{\beta, j}^{\prime}-\sum_{i=0}^{N} \chi_{N, i}^{\prime} w_{\beta, j}^{*}\left(\cdot ; y_{N, i}\right)\right\|_{L^{\infty}(\Omega \times \mathcal{S})} \\
& =\left\|\mathcal{J}_{N, \beta, j}^{\prime}-\sum_{i=0}^{N} \chi_{N, i}^{\prime} w_{\beta, j}^{*}\left(\cdot ; y_{N, i}\right)+\mathcal{J}_{\beta, j}^{\prime}-\mathcal{J}_{N, \beta, j}^{\prime}\right\|_{L^{\infty}(\Omega \times \mathcal{S})} \\
& \leq\left\|\mathcal{J}_{N, \beta, j}^{\prime}-\sum_{i=0}^{N} \chi_{N, i}^{\prime} w_{\beta, j}^{*}\left(\cdot ; y_{N, i}\right)\right\|_{L^{\infty}(\Omega \times \mathcal{S})}+\left\|\mathcal{J}_{\beta, j}^{\prime}-\mathcal{J}_{N, \beta, j}^{\prime}\right\|_{L^{\infty}(\Omega \times \mathcal{S})} .
\end{aligned}
$$

Here, $\mathcal{J}_{N, \beta, j}^{\prime} \equiv\left(\mathcal{J}_{N, \beta, j}\right)^{\prime}=\sum_{i=0}^{N} \mathcal{J}_{\beta, j}\left(\cdot ; y_{N, i}\right) \chi_{N, i}^{\prime}(\cdot)$.

We first develop a bound for the first term on the right hand side of (37). By (35) and the triangle inequality we obtain

$$
\begin{aligned}
& \left\|\mathcal{J}_{N, \beta, j}^{\prime}-\sum_{i=0}^{N} \chi_{N, i}^{\prime} w_{\beta, j}^{*}\left(\cdot ; y_{N, i}\right)\right\|_{L^{\infty}(\Omega \times \mathcal{S})} \\
& =\left\|\sum_{i=0}^{N}\left(\mathcal{J}_{\beta, j}\left(\cdot ; y_{N, i}\right)-w_{\beta, j}^{*}\left(\cdot ; y_{N, i}\right)\right) \chi_{N, i}^{\prime}\right\|_{L^{\infty}(\Omega \times \mathcal{S})} \\
& \leq\left\|\sum_{i=0}^{N}\left|\chi_{N, i}^{\prime}\right|\right\| \mathcal{J}_{\beta, j}\left(\cdot ; y_{N, i}\right)-w_{\beta, j}^{*}\left(\cdot ; y_{N, i}\right) \mid \|_{L^{\infty}(\Omega \times \mathcal{S})} \\
& \leq\left\|\max _{0 \leq i \leq N}\left|\mathcal{J}_{\beta, j}\left(\cdot ; y_{N, i}\right)-w_{\beta, j}^{*}\left(\cdot ; y_{N, i}\right)\right| \sum_{j=0}^{N}\left|\chi_{N, j}^{\prime}\right|\right\|_{L^{\infty}(\Omega \times \mathcal{S})}
\end{aligned}
$$

\footnotetext{
${ }^{2}$ Note that $w_{\beta, j}^{*}$ depends on all $P$ parameter values $\mu_{(i)}, 1 \leq i \leq P$. However we shall suppress the dependence on parameters $\mu_{(i)}, i \neq j$, for simplicity of notation.
} 
Further, by Hypothesis 2, by taking the maximum over the interval $\mathcal{S}$, by the definition of $w_{\beta, j}^{*}$ in (36), and finally the definition of $e_{M}^{p}$ in (32), we obtain

$$
\begin{aligned}
\left\|\max _{0 \leq i \leq N}\left|\mathcal{J}_{\beta, j}\left(\cdot ; y_{N, i}\right)-w_{\beta, j}^{*}\left(\cdot ; y_{N, i}\right)\right| \sum_{j=0}^{N}\left|\chi_{N, j}^{\prime}\right|\right\|_{L^{\infty}(\Omega \times \mathcal{S})} \\
\quad \leq D(N) \max _{0 \leq i \leq N}\left\|\mathcal{J}_{\beta, j}\left(\cdot ; y_{N, i}\right)-w_{\beta, j}^{*}\left(\cdot ; y_{N, i}\right)\right\|_{L^{\infty}(\Omega)} \\
\quad \leq D(N) \max _{y \in \mathcal{S}}\left\|\mathcal{J}_{\beta, j}(\cdot ; y)-w_{\beta, j}^{*}(\cdot ; y)\right\|_{L^{\infty}(\Omega)} \\
\quad=D(N) \max _{y \in \mathcal{S}} \inf _{w \in W_{M}}\left\|\mathcal{J}_{\beta, j}(\cdot ; y)-w\right\|_{L^{\infty}(\Omega)} \\
\quad \leq D(N) e_{M}^{p}
\end{aligned}
$$

and hence

$$
\left\|\mathcal{J}_{N, \beta, j}^{\prime}-\sum_{i=0}^{N} \chi_{N, i}^{\prime} w_{\beta, j}^{*}\left(\cdot ; y_{N, i}\right)\right\|_{L^{\infty}(\Omega \times \mathcal{S})} \leq D(N) e_{M}^{p}
$$

We next develop a bound for the second term on the right hand side of (37). To this end we invoke the fact that for any $x \in \Omega$ the function $\mathcal{J}_{\beta, j}(x ; \cdot)$ belongs to the family $\mathcal{H}$ defined by (33). We may thus invoke Hypothesis 1 to directly obtain

$$
\left\|\mathcal{J}_{\beta, j}^{\prime}(x ; \cdot)-\left(I_{N} \mathcal{J}_{\beta, j}(x ; \cdot)\right)^{\prime}\right\|_{L^{\infty}(\Omega \times \mathcal{S})} \leq G(N) .
$$

We may now combine (37) with (47) and (48) to obtain

$$
\left\|\mathcal{J}_{\beta, j}^{\prime}-\sum_{i=0}^{N} \chi_{N, i}^{\prime} w_{\beta, j}^{*}\left(\cdot ; y_{N, i}\right)\right\|_{L^{\infty}(\Omega \times \mathcal{S})} \leq G(N)+D(N) e_{M}^{p}
$$

Next, we introduce $\beta_{j}^{+}=\beta+e_{j}$ where $e_{j}$ is the canonical unit vector of dimension $P$ with the $j$ 'th entry equal to unity; we recall that $\beta$ has length $|\beta|=p$ and hence $\beta_{j}^{+}$has length $\left|\beta_{j}^{+}\right|=p+1$. We note that the multi-index $\beta$, the parameter values $\mu_{(i)} \in \mathcal{D}_{(i)}, 1 \leq i \leq P, i \neq j$, as well as the dimension $j$, were chosen arbitrarily above. We may thus conclude (recall above we wrote $y=\mu_{(j)}$ for each fixed $j$ ) that ${ }^{3}$

$$
\begin{array}{r}
\max _{\beta \in \mathcal{M}_{p}} \max _{1 \leq j \leq P} \max _{\mu \in \mathcal{D}}\left\|\mathcal{F}^{\left(\beta_{j}^{+}\right)}(\cdot ; \mu)-\sum_{i=0}^{N} \chi_{N, i}^{\prime}\left(\mu_{(j)}\right) w_{\beta, j}^{*}\left(\cdot ; y_{N, i}\right)\right\|_{L^{\infty}(\Omega)} \\
\leq G(N)+D(N) e_{M}^{p}
\end{array}
$$

We note that for any $\beta \in \mathcal{M}_{p}$, any $\mu_{(j)} \in \mathcal{D}_{(j)}$, and any $1 \leq j \leq P$, the function $\sum_{i=0}^{N} \chi_{N, i}^{\prime}\left(\mu_{(j)}\right) w_{\beta, j}^{*}\left(\cdot ; y_{N, i}\right)$ is just one particular member of $W_{M}$. For

\footnotetext{
${ }^{3}$ Recall that $w_{\beta, j}^{*}$ depends implicitly on the parameter values $\mu_{(i)}, i \neq j$.
} 
the error $e_{M}^{p+1}$ of the best approximation of any derivative of order $p+1$ in $W_{M}$ we thus obtain

$$
e_{M}^{p+1}=\max _{\beta \in \mathcal{M}_{p+1}} \max _{\mu \in \mathcal{D}} \inf _{w \in W_{M}}\left\|\mathcal{F}^{(\beta)}(\cdot ; \mu)-w\right\|_{L^{\infty}(\Omega)} \leq G(N)+D(N) e_{M}^{p} .
$$

The final step is to bound the right-hand side of (51) in terms of $e_{M}^{p}$ alone. To this end we note that we may choose $N$ freely. In particular we may choose $N$ as the minimizer of the right hand side of (51). However for simplicity we shall make a different choice for $N$; in particular we choose $N=N_{\text {bal }}\left(e_{M}^{p}\right)$ to balance the two terms on the right hand side of (51). With this choice we obtain

$$
e_{M}^{p+1} \leq 2 D\left(N_{\text {bal }}\left(e_{M}^{p}\right)\right) e_{M}^{p}=2 H\left(e_{M}^{p}\right),
$$

and thus $e_{M}^{p+1} \rightarrow 0$ as $e_{M}^{p} \rightarrow 0$ by Hypothesis 3 .

We now provide three lemmas, each of which quantifies the convergence in Proposition 1. The first lemma quantifies the convergence in Proposition 1 in the case that $\mathcal{F}(x ; \cdot) \in C^{1}(\mathcal{D})$ for all $x \in \Omega$.

Lemma 1. Assume for all $x \in \Omega$ that $\mathcal{F}^{(\beta)}(x ; \cdot) \in C^{1}(\mathcal{D})$ and furthermore that all second order derivatives of $\mathcal{F}^{(\beta)}(x ; \cdot)$ are bounded over $\mathcal{D}$. Then for any fixed $p=|\beta| \geq 0$ there is a constant $C_{p+1}>0$ (independent of $M$ ) such that for any $M$

$$
e_{M}^{p+1} \leq C_{p+1} \sqrt{e_{M}^{p}}
$$

Proof. In this case we may invoke piecewise linear interpolation as our interpolation system in the proof of Proposition 1. By (12) and (52) we obtain $e_{M}^{p+1} \leq 2\left(c_{\mathcal{H}}^{\operatorname{lin}} e_{M}^{p}\right)^{1 / 2}$. The result follows for $C_{p+1}=2\left(c_{\mathcal{H}}^{\operatorname{lin}}\right)^{1 / 2}$.

The next lemma quantifies the convergence in Proposition 1 in the case that $\mathcal{F}^{(\beta)}(x ; \cdot) \in C^{2}(\mathcal{D})$ for all $x \in \Omega$.

Lemma 2. Assume for all $x \in \Omega$ that $\mathcal{F}^{(\beta)}(x ; \cdot) \in C^{2}(\mathcal{D})$ and furthermore that all third order derivatives of $\mathcal{F}^{(\beta)}(x ; \cdot)$ are bounded over $\mathcal{D}$. Then for any fixed $p=|\beta| \geq 0$ there is a constant $C_{p+1}>0$ (independent of $M$ ) such that for any $M$

$$
e_{M}^{p+1} \leq C_{p+1}\left(e_{M}^{p}\right)^{2 / 3} .
$$

Proof. In this case we may invoke piecewise quadratic interpolation as our interpolation system in the proof of Proposition 1. By (19) and (52) we obtain $e_{M}^{p+1} \leq$ $5\left(2 c_{\mathcal{H}}^{\text {quad }} / 5\right)^{1 / 3}\left(e_{M}^{p}\right)^{2 / 3}$. The result follows for $C_{p+1}=5\left(2 c_{\mathcal{H}}^{\text {quad }} / 5\right)^{1 / 3}$.

We make the following remark concerning Lemma 1 and Lemma 2 in the case of algebraic convergence. 
Remark 1. Let $|\beta|=p$, and assume for all $x \in \Omega$ that $\mathcal{F}^{(\beta)}(x, \cdot) \in C^{q_{p}}(\mathcal{D})$, $q_{p}>0$, and furthermore that all $q_{p}+1$ order derivatives of $\mathcal{F}^{(\beta)}(x, \cdot)$ are bounded over $\mathcal{D}$. Suppose in this case that for all $M$ we have $e_{M}^{p} \leq$ const $\cdot M^{-r_{p}}$ with $r_{p}>0 .{ }^{4}$ For $q_{p}=1$ we may invoke Lemma 1 to obtain

$$
e_{M}^{p+1} \leq C_{p+1}\left(e_{M}^{p}\right)^{\frac{1}{2}} \propto M^{-\frac{r_{p}}{2}}=M^{\frac{r_{p}}{2}} e_{M}^{p} .
$$

Similarly, for $q_{p}=2$ we may invoke Lemma 2 to obtain

$$
e_{M}^{p+1} \leq C_{p+1}\left(e_{M}^{p}\right)^{\frac{2}{3}} \propto M^{-\frac{2 r_{p}}{3}}=M^{\frac{r_{p}}{3}} e_{M}^{p} .
$$

More generally, with higher-regularity versions of Lemma 1 and Lemma 2, we expect for any $q_{p}>0$ that

$$
e_{M}^{p+1} \leq C_{p+1}\left(e_{M}^{p}\right)^{1-\frac{1}{q_{p}}} \propto M^{-r_{p}\left(1-\frac{1}{q_{p}}\right)}=M^{\frac{r_{p}}{q_{p}+1}} e_{M}^{p} .
$$

for any $q_{p}>0$. We shall comment on these estimates further in our discussion of numerical results in Section 5.

The third lemma quantifies the convergence in Proposition 1 in the case that $\mathcal{F}(x, \cdot)$ is analytic over $\mathcal{D}$.

Lemma 3. Assume for all $x \in \Omega$ that $\mathcal{F}(x, \cdot): \mathcal{D} \rightarrow \mathbb{R}$ is analytic over $\mathcal{D}$. Then for any fixed $p \geq 0$ there exists a constant $C_{p+1}>0$ (independent of $M$ ) such that for any $M>M_{0}$,

$$
e_{M}^{p+1} \leq C_{p+1} \log \left(e_{M}^{p}\right)^{2} e_{M}^{p} .
$$

In particular, if for some $p$ (independent of $M$ )

$$
e_{M}^{p} \leq \hat{c} M^{\sigma} e^{-\gamma M^{\alpha}}
$$

where $\sigma$ and $\alpha$ are constants and $\gamma$ and $\hat{c}$ are positive constants, then there exists a constant $\hat{C}_{p+1}$ such that

$$
e_{M}^{p+1} \leq \hat{C}_{p+1} M^{\sigma+2 \alpha} e^{-\gamma M^{\alpha}} .
$$

Proof. In this case we may invoke Chebyshev interpolation as our interpolation system in the proof of Proposition 1. For suffiently large $M$ (i.e. sufficiently small $e_{M}^{p}$ ), we may use (24) and (52) to obtain $e_{M}^{p+1}<2 A^{2}\left(\log \left(1 / e_{M}^{p}\right)\right)^{2} e_{M}^{p}$. The result (58) follows for $C_{p+1}=2 A^{2}$ since $\left(\log \left(1 / e_{M}^{p}\right)\right)^{2}=\left(\log \left(e_{M}^{p}\right)\right)^{2}$.

The additional result (60) follows from the assumption (59) since the right hand side of (58) decreases monotonically as $e_{M}^{p} \rightarrow 0$ for $e_{M}^{p}<e^{-2}$. We obtain in this case

$$
\begin{aligned}
& e_{M}^{p+1} \leq C_{p+1} \log \left(\hat{c} M^{\sigma} e^{-\gamma M^{\alpha}}\right)^{2} M^{\sigma} e^{-\gamma M^{\alpha}} \\
& =C_{p+1}\left(\log \hat{c}+\sigma \log M-\gamma M^{\alpha}\right)^{2} M^{\sigma} e^{-\gamma M^{\alpha}} \\
& \leq \hat{C}_{p+1} M^{2 \alpha} M^{\sigma} e^{-\gamma M^{\alpha}}
\end{aligned}
$$

for $M$ sufficiently large.

\footnotetext{
${ }^{4}$ The convergence rate $r_{p}$ will depend on the sequence of spaces $W_{M}$; we expect that the convergence rate also will depend on the parametric regularity $q_{p}$.
} 
We make the following two remarks concerning Lemma 3.

Remark 2. Note that Lemma 3, in contrast to 1 and 2, only holds for all $M$ sufficiently large. The reason is that to obtain (58) we invoke the assumption $\mathcal{W}(\xi)<\log (\xi)$, which holds only for $\xi>e$ (see Appendix A.3 for a proof). Since here $\xi \propto 1 / e_{M}^{p}$, this assumption will be satisfied when $e_{M}$ is sufficiently small, i.e. when $M$ is sufficiently large.

Remark 3. We note that we may invoke the result (60) recursively to obtain, for any fixed $p$ and all $M>\hat{M}_{0, p}$,

$$
\epsilon_{M}^{p} \leq \tilde{C}_{p} M^{\sigma+2 \alpha p} e^{-\gamma M}
$$

whenever $\epsilon_{M}^{0} \leq c M^{\sigma} e^{-\gamma M^{\alpha}}$. Here, $\tilde{C}_{p}$ and $\hat{M}_{0, p}$ depend on $p$, but $\tilde{C}_{p}$ does not depend on $M$.

\section{The Empirical Interpolation Method}

In this section we first recall the Empirical Interpolation Method (EIM) [1, 8, 9] and then consider the convergence theory of the previous section applied to the EIM. The EIM approximation space is spanned by precomputed snapshots of a parameter dependent "generating function" for judiciously chosen parameter values from a predefined parameter domain. Given any new parameter value in this parameter domain, we can construct an approximation to the generating function at this new parameter value - or in principle an approximation to any function defined over the same spatial domain - as a linear combination of the EIM basis functions. The particular linear combination is determined through interpolation at judiciously chosen points in the spatial domain. For parametrically smooth functions, the EIM approximation to the generating function yields rapid — typically exponential — convergence.

\subsection{Procedure}

We introduce the generating function $\mathcal{G}: \Omega \times \mathcal{D} \rightarrow \mathbb{R}$ such that for all $\mu \in \mathcal{D}$, $\mathcal{G}(\cdot ; \mu) \in L^{\infty}(\Omega)$. We introduce a training set $\Xi_{\text {train }} \subset \mathcal{D}$ of finite cardinality $\left|\Xi_{\text {train }}\right|$ which shall serve as our computational surrogate for $\mathcal{D}$. We also introduce a triangulation $\mathcal{T}_{\mathcal{N}}(\Omega)$ of $\Omega$ with $\mathcal{N}$ vertices over which we shall in practice, for any $\mu \in \mathcal{D}$, realize $\mathcal{G}(\cdot ; \mu)$ as a piecewise linear function.

Now, for $1 \leq M \leq M_{\max }<\infty$, we define the EIM approximation space $W_{M}^{\mathcal{G}}$ and the EIM interpolation nodes $T_{M}^{\mathcal{G}}$ associated with $\mathcal{G}$; here, $M_{\max }$ is a specified maximum EIM appproximation space dimension. We first choose (randomly, say) an initial parameter value $\mu_{1} \in \mathcal{D}$; we then determine the first EIM interpolation node as $t_{1}=\arg \sup _{x \in \Omega}\left|\mathcal{G}\left(x ; \mu_{1}\right)\right|$; we next define the first EIM basis function as $q_{1}=\mathcal{G}\left(\cdot ; \mu_{1}\right) / \mathcal{G}\left(t_{1} ; \mu_{1}\right)$. We can then, for $M=1$, define $W_{M}^{\mathcal{G}}=\operatorname{span}\left\{q_{1}\right\}$ and $T_{M}^{\mathcal{G}}=\left\{t_{1}\right\}$. We also define a nodal value matrix $B^{1}$ with (a single) element $B_{1,1}^{1}=q_{1}\left(t_{1}\right)=1$. 
Next, for $2 \leq M \leq M_{\max }$, we first compute the empirical interpolation of $\mathcal{G}(\cdot ; \mu)$ for all $\mu \in \Xi_{\text {train }}$ : we solve the linear system

$$
\sum_{j=1}^{M-1} \phi_{j}^{M-1}(\mu) B_{i, j}^{M-1}=\mathcal{G}\left(t_{i} ; \mu\right), \quad 1 \leq i \leq M-1,
$$

and compute the empirical interpolation $\mathcal{G}_{M-1}(\cdot ; \mu) \in W_{M-1}^{\mathcal{G}}$ as

$$
\mathcal{G}_{M-1}(\cdot ; \mu)=\sum_{i=1}^{M-1} \phi_{i}^{M-1}(\mu) q_{i}
$$

for all $\mu \in \Xi_{\text {train }}$. We then choose the next parameter $\mu_{M} \in \mathcal{D}$ as the maximizer of the EIM interpolation error over the training set,

$$
\mu_{M}=\arg \max _{\mu \in \Xi_{\text {train }}}\left\|\mathcal{G}_{M-1}(\cdot ; \mu)-\mathcal{G}(\cdot ; \mu)\right\|_{L^{\infty}(\Omega)} ;
$$

note that thanks to our piecewise linear realization of $\mathcal{G}(\cdot ; \mu)$, the norm evaluation is a simple comparison of function values at the $\mathcal{N}$ vertices of $\mathcal{T}_{\mathcal{N}}(\Omega)$. We now choose the next EIM interpolation node as the point in $\Omega$ at which the EIM error associated with $\mathcal{G}_{M-1}\left(\mu_{M}\right)$ is largest,

$$
t_{M}=\arg \sup _{x \in \Omega}\left|\mathcal{G}_{M-1}\left(x ; \mu_{M}\right)-\mathcal{G}\left(x ; \mu_{M}\right)\right| .
$$

The next EIM basis function is then

$$
q_{M}=\frac{\mathcal{G}_{M-1}\left(\cdot ; \mu_{M}\right)-\mathcal{G}\left(\cdot ; \mu_{M}\right)}{\mathcal{G}_{M-1}\left(t_{M} ; \mu_{M}\right)-\mathcal{G}\left(t_{M} ; \mu_{M}\right)} .
$$

We finally enrich the EIM space: $W_{M}^{\mathcal{G}}=\operatorname{span}\left\{q_{1}, \ldots, q_{M}\right\}$; expand the set of nodes: $T_{M}^{\mathcal{G}}=\left\{t_{1}, \ldots, t_{M}\right\}$; and expand the nodal value matrix: $B_{i, j}^{M}=q_{j}\left(t_{i}\right)$, $1 \leq i, j \leq M$.

Now, given any function $\mathcal{F}: \Omega \times \mathcal{D} \rightarrow \mathbb{R}$ (in particular, we shall consider $\left.\mathcal{F}=\mathcal{G}^{(\beta)}\right)$, we define for any $\mu \in \mathcal{D}$ and for $1 \leq M \leq M_{\max }$ the empirical interpolation of $\mathcal{F}(\cdot ; \mu)$ in the space $W_{M}^{\mathcal{G}}$ (the space generated by $\mathcal{G}$ ) as

$$
\mathcal{F}_{M}^{\mathcal{G}}(\cdot ; \mu)=\sum_{i=1}^{M} \phi_{i}^{M}(\mu) q_{i},
$$

where the coefficients $\phi_{i}^{M}(\mu), 1 \leq i \leq M$, solve the linear system

$$
\sum_{j=1}^{M} \phi_{j}^{M}(\mu) B_{i, j}^{M}=\mathcal{F}\left(t_{i} ; \mu\right), \quad 1 \leq i \leq M .
$$

We note that by construction the matrices $B^{M} \in \mathbb{R}^{M \times M}, 1 \leq M \leq M_{\max }$, are lower triangular: by $(63), \mathcal{G}_{M-1}\left(t_{j} ; \mu_{M}\right)=\mathcal{G}\left(t_{j} ; \mu_{M}\right)$ for $j<M$. As a result, 
computation of the EIM coefficients $\phi_{j}^{M}, 1 \leq j \leq M$, in (69) and (63) are $\mathcal{O}\left(M^{2}\right)$ operations. We emphasize that the computational cost associated with the EIM approximation (68)-(69) (after snapshot precomputation) is independent of the number $\mathcal{N}$ of vertices in the triangulation $\mathcal{T}_{\mathcal{N}}(\Omega)$. We may thus choose $\mathcal{N}$ conservatively.

We next note that, for any multi-index $\beta$,

$$
\left(\mathcal{F}_{M}^{\mathcal{G}}\right)^{(\beta)}=\left(\sum_{i=1}^{M} \phi_{i}^{M}(\mu) q_{i}\right)^{(\beta)}=\sum_{i=1}^{M} \varphi_{i}^{M}(\mu) q_{i}
$$

where $\varphi_{i}^{M}(\mu)=\left(\phi_{i}^{M}\right)^{(\beta)}(\mu), 1 \leq i \leq M$, solve the linear system (recall that the matrix $B^{M}$ is $\mu$-independent)

$$
\sum_{j=1}^{M} \varphi_{j}^{M}(\mu) B_{i, j}^{M}=\mathcal{F}^{(\beta)}\left(t_{i} ; \mu\right), \quad 1 \leq i \leq M .
$$

Hence,

$$
\left(\mathcal{F}_{M}^{\mathcal{G}}\right)^{(\beta)}=\left(\mathcal{F}^{(\beta)}\right)_{M}^{\mathcal{G}},
$$

that is, the parametric derivative of the approximation is equivalent to the approximation of the parametric derivative. We note that this equivalence holds since we invoke the same approximation space $W_{M}^{\mathcal{G}}$ for both EIM approximations $\mathcal{F}_{M}^{\mathcal{G}}$ and $\left(\mathcal{F}^{(\beta)}\right)_{M}^{\mathcal{G}}$.

\subsection{Convergence theory applied to the EIM}

We introduce the Lebesgue constants [10]

$$
\Lambda_{M}=\sup _{x \in \Omega} \sum_{i=1}^{M}\left|V_{i}^{M}(x)\right|, \quad 1 \leq M \leq M_{\max },
$$

where $V_{i}^{M} \in W_{M}^{\mathcal{G}}$ are the characteristic functions associated with $W_{M}^{\mathcal{G}}$ and $T_{M}^{\mathcal{G}}$ : $V_{i}^{M}\left(t_{j}\right)=\delta_{i, j}, 1 \leq i, j \leq M$. Our theory of Section 3 considers the convergence in the best approximation error. However, we can relate the EIM approximation of $\mathcal{F}$ to the best approximation of $\mathcal{F}$ through

Lemma 4. The error in the EIM derivative approximation satisfies

$$
\begin{aligned}
& \left\|\mathcal{F}^{(\beta)}(\cdot ; \mu)-\left(\mathcal{F}^{(\beta)}\right)_{M}^{\mathcal{G}}(\cdot ; \mu)\right\|_{L^{\infty}(\Omega)} \\
& \quad \leq\left(1+\Lambda_{M}\right) \inf _{w \in W_{M}^{\mathcal{G}}}\left\|\mathcal{F}^{(\beta)}(\cdot ; \mu)-w\right\|_{L^{\infty}(\Omega)} .
\end{aligned}
$$

Proof. The proof is identical to [1, Lemma 3.1]. We first introduce $\mathcal{F}_{M}^{\mathcal{G}, *}(\cdot ; \mu)=$ $\arg \inf _{w \in W_{M}^{\mathcal{G}}}\|\mathcal{F}(\cdot ; \mu)-w\|_{L^{\infty}(\Omega)}$, and define coefficient functions $\omega_{m}^{M}(\mu), 1 \leq$ 
$m \leq M$, such that $\mathcal{F}_{M}^{\mathcal{G}}(\cdot ; \mu)-\mathcal{F}_{M}^{\mathcal{G}, *}(\cdot ; \mu)=\sum_{m=1}^{M} \omega_{m}^{M}(\mu) q_{m}$. By the interpolation property of the EIM we then obtain

$$
\mathcal{F}\left(t_{n} ; \mu\right)-\mathcal{F}_{M}^{\mathcal{G}, *}\left(t_{n} ; \mu\right)=\mathcal{F}_{M}^{\mathcal{G}}\left(t_{n} ; \mu\right)-\mathcal{F}_{M}^{\mathcal{G}, *}\left(t_{n} ; \mu\right)=\sum_{m=1}^{M} \omega_{m}^{M}(\mu) q_{m}\left(t_{n}\right) .
$$

We then introduce $E_{M}^{\mathcal{G}}(\mu)=\left\|\mathcal{F}(\cdot ; \mu)-\mathcal{F}_{M}^{\mathcal{G}}(\cdot ; \mu)\right\|_{L^{\infty(\Omega)}}$ and $E_{M}^{\mathcal{G}, *}(\mu)=\| \mathcal{F}(\cdot ; \mu)-$ $\mathcal{F}_{M}^{\mathcal{G}, *}(\cdot ; \mu) \|_{L^{\infty}(\Omega)}$ and note that

$$
\begin{gathered}
E_{M}^{\mathcal{G}}(\mu)-E_{M}^{\mathcal{G}, *}(\mu) \leq\left\|\sum_{m=1}^{M} \omega_{m}^{M}(\mu) q_{m}\right\|_{L^{\infty}(\Omega)}=\left\|\sum_{n=1}^{M} \sum_{m=1}^{M} \omega_{m}^{M}(\mu) q_{m}\left(t_{n}\right) V_{n}^{M}\right\|_{L^{\infty}(\Omega)} \\
=\left\|\sum_{n=1}^{M}\left(\mathcal{F}\left(t_{n} ; \mu\right)-\mathcal{F}_{M}^{\mathcal{G}, *}\left(t_{n} ; \mu\right)\right) V_{n}^{M}\right\|_{L^{\infty}(\Omega)} \leq \Lambda_{M} E_{M}^{\mathcal{G}, *}(\mu)
\end{gathered}
$$

since $\left|\mathcal{F}\left(t_{n} ; \mu\right)-\mathcal{F}_{M}^{\mathcal{G}, *}\left(t_{n} ; \mu\right)\right| \leq E_{M}^{\mathcal{G}, *}(\mu), 1 \leq n \leq M$, and by the definition of $\Lambda_{M}$ in (73). The result (74) follows for any $\beta$ by replacing $\mathcal{F}$ by $\mathcal{F}^{(\beta)}$ in the arguments above.

It can be proven $[1,8]$ that $\Lambda_{M}<2^{M}-1$. However, in actual practice the growth of $\Lambda_{M}$ is much slower than this exponential upper bound, as we shall observe below (see also results in $[1,8,9]$ ). Based on Lemma 4 and the anticipated slow growth of $\Lambda_{M}$, we expect the EIM approximation to any parametric derivative to be good as long as the best approximation is good.

\section{$5 \quad$ Numerical Results}

In this section we demonstrate the theory through two numerical examples. In each example, we consider a parametrized function $\mathcal{F}$ and we generate approximation spaces with the EIM method for $\mathcal{G}=\mathcal{F}$ as the generating function. To confirm the theory we compute for a large number of parameter values in a test set $\Xi_{\text {test }} \subset \mathcal{D}, \Xi_{\text {test }} \neq \Xi_{\text {train }}$, the best approximation of $\mathcal{F}$ and the parametric derivatives $\mathcal{F}^{(\beta)}$ in these spaces. We define the maximum best approximation error over the space of dimension $M$ as

$$
e_{M, \text { test }}^{p} \equiv \max _{\beta \in \mathcal{M}_{p}} \max _{\mu \in \Xi_{\text {test }}} \inf _{w \in W_{M}^{\mathcal{G}}}\left\|\mathcal{F}^{(\beta)}(\cdot ; \mu)-w\right\|_{L^{\infty}(\Omega)}
$$

(the test set $\Xi_{\text {test }}$ will be different for each example problem). We note that thanks to the piecewise linear representation of $\mathcal{F}$ (and its parametric derivatives), determination of the best approximation (and associated error) is equivalent to the solution of a linear program for each $\mu \in \Xi_{\text {test }}$.

We shall also compute error degradation factors

$$
\rho_{M . \text { test }}^{p} \equiv \frac{e_{M, \text { test }}^{p}}{e_{M, \text { test }}^{0}}
$$




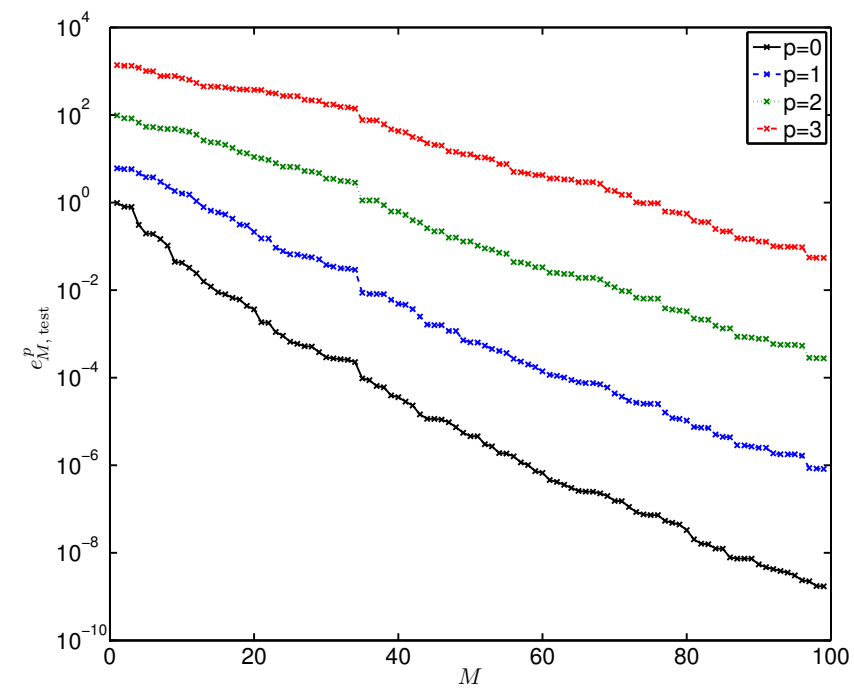

Figure 1: The maximum $L^{\infty}(\Omega)$ projection error over the test set, $e_{M \text {,test }}^{p}$, for $0 \leq p \leq 3$ for Example 1 .

as a measure of how much accuracy we loose for different differential order $p$ of the function to be approximated.

We finally confirm for each example that the growth of the Lebesgue constant is only modest and hence that, by Lemma 4, the EIM approximation will be close to the best approximation.

\subsection{Example 1: Parametrically smooth Gaussian surface}

We introduce the spatial domain $\Omega=[0,1]^{2}$ and the parameter domain $\mathcal{D}=$ $[0.4,0.6]^{2}$. We consider the $2 \mathrm{D}$ Gaussian $\mathcal{F}: \Omega \times \mathcal{D} \rightarrow \mathbb{R}$ defined by

$$
\mathcal{F}(x ; \mu)=\exp \left(\frac{-\left(x_{(1)}-\mu_{(1)}\right)^{2}-\left(x_{(2)}-\mu_{(2)}\right)^{2}}{2 \sigma^{2}}\right)
$$

for $x \in \Omega, \mu \in \mathcal{D}$, and $\sigma \equiv 0.1$. This function is thus parametrized by the location of the maximum of the Gaussian surface. We note that for all $x \in \Omega$ the function $\mathcal{F}(x ; \cdot)$ is analytic over $\mathcal{D}$; we may thus invoke Lemma 3.

We introduce a triangulation $\mathcal{T}_{\mathcal{N}}(\Omega)$ with $\mathcal{N}=2601$ vertices; we introduce an equi-distant training set "grid" $\Xi_{\text {train }} \subset \mathcal{D}$ of size $\left|\Xi_{\text {train }}\right|=900=30 \times 30$. We then pursue the EIM with $\mathcal{G}=\mathcal{F}$ for $M_{\max }=99$.

We now introduce a uniformly distributed random test set $\Xi_{\text {test }} \subset \mathcal{D}$ of size 1000 over which we compute best approximation errors $e_{M}^{p}, 1 \leq M \leq$ $M_{\max }$. In Figure 1 we show the maximum best approximation errors $e_{M \text {,test }}^{p}$ for $p=0,1,2,3$. We note that the convergence is exponential not only for the best approximation of $\mathcal{F}(p=0)$, but also for the best approximation of its 


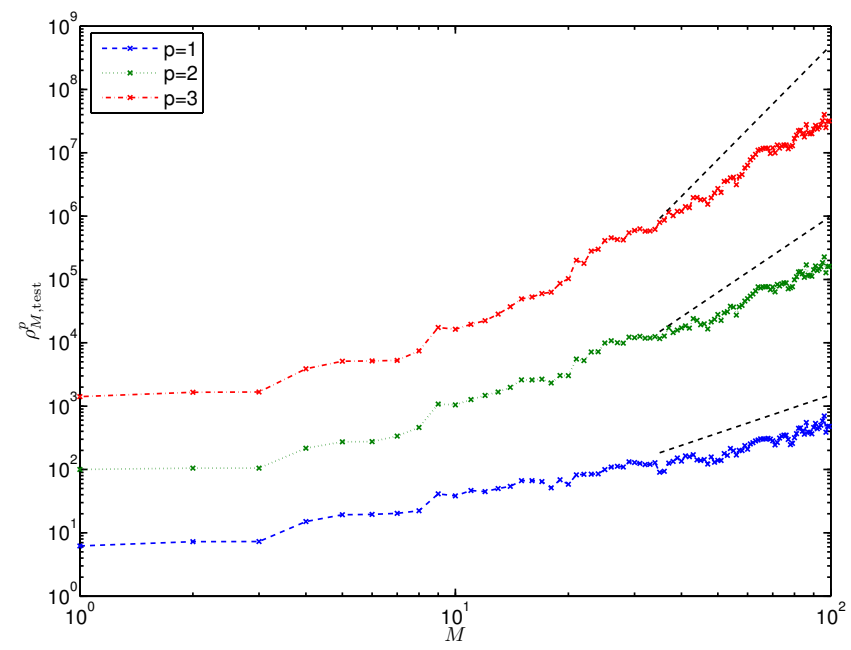

Figure 2: Error degradation factors $\rho_{M \text {,test }}^{p}, p=1,2,3$, for Example 1. The shorter dashed lines are of slope $M^{2 p}$.

derivatives $(p>0)$. We also note that for large $M$, the (exponential) rates of convergence associated with the parametric derivatives are close to the rate associated with the generating function.

To provide for some theoretical explanation for these observations we make the assumption $e_{M}^{0}=\hat{c} M^{\sigma} e^{-\gamma M}$. An ordinary least squares linear regression on $\log \left(e_{M}^{0}\right)$ for $35 \leq M \leq M_{\max }$ provides estimates $\log \hat{c} \approx 4.4194, \sigma \approx-4.4611$, and $\gamma \approx 0.0436$. Based on these estimates and the relatively small associated standard errors ${ }^{5}$ we may expect that this assumption holds. In this case, we expect from Remark 3 that $e_{M}^{p} \leq \tilde{C}_{p} M^{\sigma+2 p} e^{-\gamma M}$ also for $p>0$. This result thus explains the exponential convergence associated with the parametric derivatives.

In Figure 2 we show the error degradation factors $\rho_{M \text {,test }}^{p}$ for $p=1,2,3$ as functions of $M$. The plot suggests that indeed $\rho_{M \text {,test }}^{p} \leq$ const $\cdot M^{2 p}$ as predicted by Remark 3 and the bounds $e_{M}^{p} \leq \tilde{C}_{p} M^{\sigma+2 p} e^{-\gamma M}, p>0$, obtained above. We note from Lemma 3 that had the result (60) been sharp we would have obtained $\rho_{M \text {,test }}^{p} \propto M^{2 p}$. We conclude that, at least for the range of $M$ considered for these computations and for this particular $\mathcal{F}$, the result (60) is not sharp.

We finally note that the factor $M^{2}$ in (60) originates from the sharp result (21); hence with our present strategy for the proof of Proposition 1 it is not clear how to sharpen (60). However, clearly our theory captures the correct qualitative behavior: we observe exponential convergence for the parametric

\footnotetext{
${ }^{5}$ For the standard errors associated with $\log \hat{c}, \sigma$, and $\gamma$ we obtain $0.6552,0.4846$, and 0.0033 , respectively. We use these standard errors as a non-rigorous measure of the uncertainty in the estimated regression parameters; however we do make particular assumptions on the regression error term and hence we can not assign any formal statistical interpretation to the standard errors.
} 


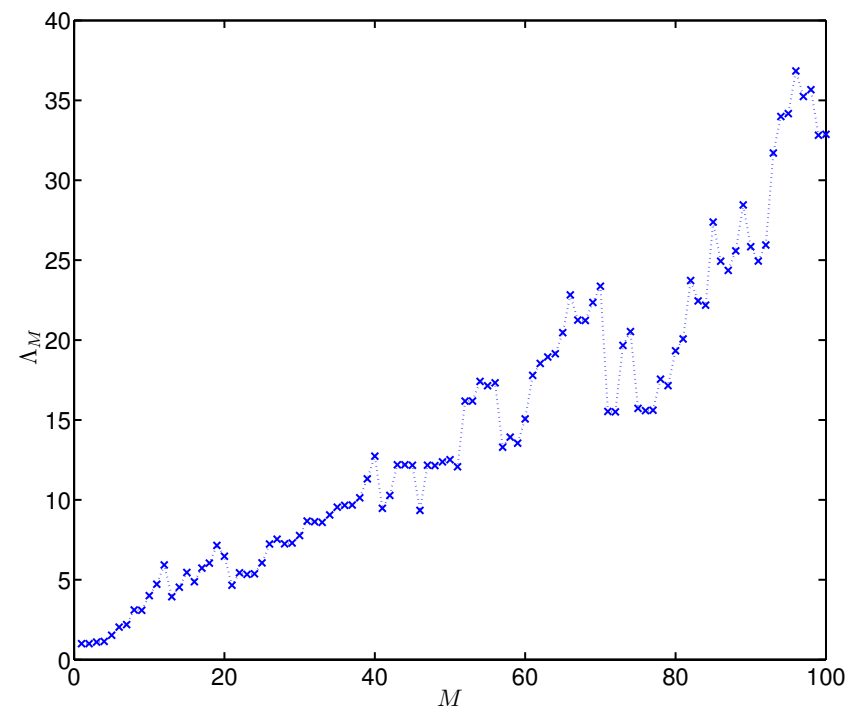

Figure 3: The Lebesgue constant $\Lambda_{M}$ for Example 1.

derivatives and there is evidence of an algebraic degradation factor for the parametric derivative approximations.

Finally, in Figure 3, we report the Lebesgue constant $\Lambda_{M}$. We note that the growth of the Lebesgue constant is only modest. The EIM derivative approximation will thus be close to the best $L^{\infty}(\Omega)$ approximation in the space $W_{M}^{\mathcal{F}}$.

\subsection{Example 2: A parametrically singular function}

We introduce the spatial domain $\Omega=[-1,1]$ and the parameter domain $\mathcal{D}=$ $[-1,1]$. We consider the function $\mathcal{F}: \Omega \times \mathcal{D} \rightarrow \mathbb{R}$ defined by

$$
\mathcal{F}(x ; \mu)=|x-\mu|^{5}
$$

for $x \in \Omega$ and $\mu \in \mathcal{D}$. The function thus has a singularity at $x=\mu$ for any $\mu \in \mathcal{D}$.

For any $x \in \Omega$ we have $\mathcal{F}^{(p)}(x ; \cdot) \in C^{q_{p}}(\mathcal{D})$ for $q_{p}=4-p$ with $\mathcal{F}^{(5)}(x ; \cdot)$ bounded over $\mathcal{D}$. Hence, to estimate $e_{M}^{1}$ from $e_{M}^{0}$, we may as indicated in Remark 1 invoke a higher order version of Lemma 1 (and Lemma 2) using piecewise quartic interpolation. Similarly, to estimate $e_{M}^{2}$ based on $e_{M}^{1}$, we may invoke a piecewise cubic version of Lemma 1 (and Lemma 2). To estimate $e_{M}^{3}$ based on $e_{M}^{2}$, we may invoke Lemma 2 directly since $\mathcal{F}^{(2)}(x ; \cdot) \in C^{2}(\mathcal{D})$ with its third order derivative bounded over $\mathcal{D}$.

We introduce a triangulation $\mathcal{T}_{\mathcal{N}}(\Omega)$ with $\mathcal{N}=500$ vertices; we introduce an equi-distant training set "grid" $\Xi_{\text {train }} \subset \mathcal{D}$ of size $\left|\Xi_{\text {train }}\right|=500$. We then pursue the EIM with $\mathcal{G}=\mathcal{F}$ for $M_{\max }=89$. 


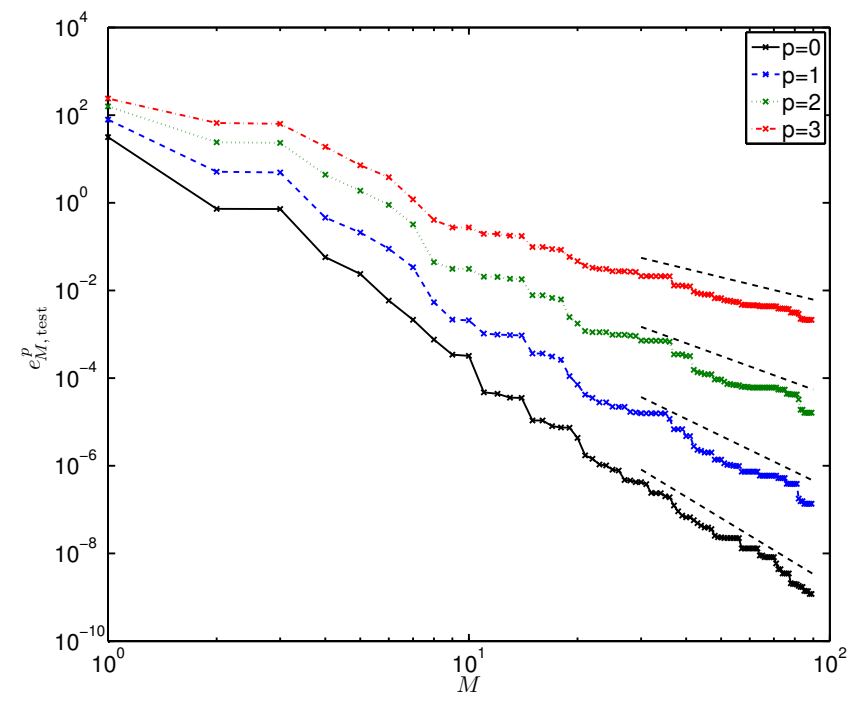

Figure 4: The maximum $L^{\infty}(\Omega)$ projection error over the test set, $e_{M \text {,test }}^{p}$, for $0 \leq p \leq 3$ for Example 2. The shorter dashed lines are of slope $M^{-5+p}$.

We now introduce a uniformly distributed random test set $\Xi_{\text {test }} \subset \mathcal{D}$ of size 500. In Figure 4 we show the maximum best approximation errors $e_{M \text {,test }}^{p}$ for $p=0,1,2,3$. The convergence is algebraic: ordinary least squares best fits to the slopes for $30 \leq M \leq M_{\max }$ yield $e_{M \text {,test }}^{0} \approx$ const $\cdot M^{-5.13}, e_{M \text {,test }}^{1} \approx$ const $\cdot M^{-4.27}, e_{M \text {,test }}^{2} \approx$ const $\cdot M^{-3.23}$, and $e_{M \text {,test }}^{3} \approx$ const $\cdot M^{-2.10}$ (the shorter dashed lines in the plot are of slope $M^{-5+p}$ ). These estimates suggest that $r_{p}=q_{p}+\omega$ where $\omega$ is somewhat larger than unity.

From Figure 4 we may also infer the approximate error degradation factors $\rho_{M \text {,test }}^{p}$ for $p=1,2,3$ as functions of $M$ : a rough estimate is $\rho_{M \text {,test }}^{p} \propto M^{p}$ since we loose approximately a factor $M$ when $p$ increases by one. We note that this is exactly what we expect from Remark 1 if $r_{p}=q_{p}+1$ and the error estimates indicated in Remark 1 are sharp.

Finally, in Figure 5, we report the Lebesgue constant $\Lambda_{M}$ : any growth of the Lebesgue constant is hardly present. The EIM derivative approximation will thus be close to the best $L^{\infty}(\Omega)$ approximation in the space $W_{M}^{\mathcal{F}}$.

\section{Concluding remarks}

We have introduced a new a priori convergence theory for the approximation of parametric derivatives. Given a sequence of approximation spaces, we have showed that the best approximation error associated with parametric derivatives of a function will go to zero provided that the best approximation error associated with the function itself goes to zero. In practice a method such as 


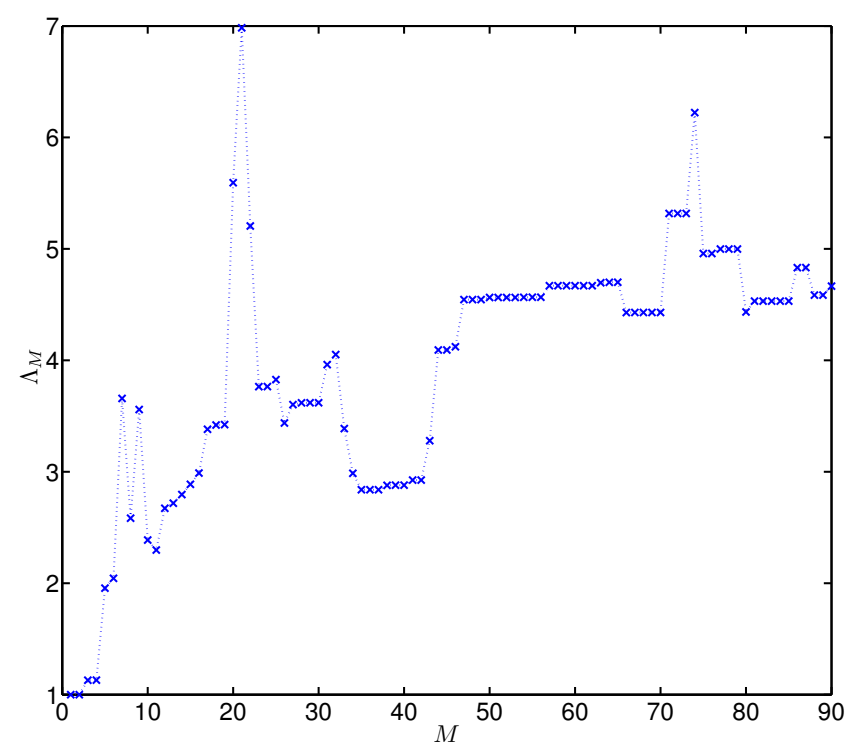

Figure 5: The Lebesgue constant $\Lambda_{M}$ for Example 2.

the EIM is used for the approximation of such functions, and hence the best approximation convergence result does not directly apply. However, thanks to the slowly growing Lebesgue constant associated with the EIM approximation scheme, we expect that the EIM approximation error will be small whenever the best approximation error is small.

A natural approach to the EIM approximation of parametric derivatives would be to either enrich the original EIM space with snapshots of these parametric derivatives or to construct separate EIM spaces for each derivative, with this derivative as the generating function. The results in this paper, however, suggest that the EIM may be invoked in practice for the approximation of parametric derivatives without enrichment of the space or construction of additional spaces.

There are admittedly several opportunities for improvements of the theory. First, our numerical results of Section 5.1 suggest that the theoretical bounds for parametrically analytic functions are not sharp. The theory predicts an error degradation factor $M^{2 p}$, but the numerical results show (for this particular example function $\mathcal{F}$ ) a smaller error degradation factor. It is not clear with the present strategy how to improve the theoretical bounds. Second, we would like to extend the validity of the theory to other (e.g. Sobolev) norms; in this case we may for example consider reduced basis [13] approximations to parametric derivatives of solutions to partial differential equations. 


\section{Acknowledgments}

This work has been partially supported by the Norwegian University of Science and Technology. The support is gratefully acknowledged.

\section{A Proofs for Hypotheses 1 and 2}

\section{A.1 Piecewise linear interpolation}

We consider piecewise linear interpolation over the equidistant interpolation nodes $y_{N, i}=(2 i / N-1) \in \Gamma=[-1,1], 0 \leq i \leq N$. In this case the characteristic functions $\chi_{N, i}$ are continuous and piecewise linear "hat functions" with support only on the interval $\left[y_{N, 0}, y_{N, 1}\right]$ for $i=0$, on $\left[y_{N, i-1}, y_{N, i+1}\right]$ for $1 \leq i \leq N-1$, and on $\left[y_{N, N-1}, y_{N, N}\right]$ for $i=N$.

We recall the results (9) and (10) from Section 2.2. Let $f: \Gamma \rightarrow \mathbb{R}$ with $f \in C^{1}(\Gamma)$ and assume that $\sup _{y \in \Gamma}\left|f^{\prime \prime}(y)\right|<\infty$. We then have, for any $y \in \Gamma$ and any $N \geq 0$,

$$
\left|f^{\prime}(y)-\left(I_{N} f\right)^{\prime}(y)\right| \leq 2 N^{-1}\left\|f^{\prime \prime}\right\|_{L^{\infty}(\Gamma)} .
$$

Further, for all $y \in \Gamma$, the characteristic functions $\chi_{N, i}, 0 \leq i \leq N$, satisfy

$$
\sum_{i=0}^{N}\left|\chi_{N, i}^{\prime}(y)\right|=N .
$$

We first demonstrate (81) (and hence (9)). For $y \in\left[y_{N, i}, y_{N, i+1}\right], 0 \leq i \leq$ $N-1$, we have

$$
\left(I_{N} f\right)^{\prime}(y)=\frac{1}{h}\left(f\left(y_{N, i+1}\right)-f\left(y_{N, i}\right)\right)
$$

where $h=2 / N$. We next write $f\left(y_{N, i}\right)$ and $f\left(y_{N, i+1}\right)$ as Taylor series around $y$ as

$$
\begin{aligned}
f\left(y_{N, i}\right) & =\sum_{j=0}^{1} \frac{f^{(j)}(x)}{j !}\left(y_{N, i}-y\right)^{j}+\int_{y}^{y_{N, i}} f^{\prime \prime}(t)\left(y_{N, i}-t\right) d t \\
f\left(y_{N, i+1}\right) & =\sum_{j=0}^{1} \frac{f^{(j)}(y)}{j !}\left(y_{N, i+1}-y\right)^{j}+\int_{y}^{y_{N, i+1}} f^{\prime \prime}(t)\left(y_{N, i+1}-t\right) d t,
\end{aligned}
$$

which we then insert in the expression $(83)$ for $\left(I_{N} f\right)^{\prime}$ to obtain

$$
\begin{gathered}
\left|\left(I_{N} f\right)^{\prime}(y)-f^{\prime}(y)\right|=\left|\frac{1}{h} \int_{y}^{y_{N, i+1}} f^{\prime \prime}(t)\left(y_{N, i+1}-t\right) d t-\frac{1}{h} \int_{y}^{y_{N, i}} f^{\prime \prime}(t)\left(y_{N, i}-t\right) d t\right| \\
\leq \frac{1}{h}\left\|f^{\prime \prime}\right\|_{L^{\infty}(\Gamma)} \max _{y \in\left[y_{N, i}, y_{N, i+1}\right]}\left(\left|y_{N, i+1}-y\right|^{2}+\left|y_{N, i}-y\right|^{2}\right) \\
\leq h\left\|f^{\prime \prime}\right\|_{L^{\infty}(\Gamma)}=2 N^{-1}\left\|f^{\prime \prime}\right\|_{L^{\infty}(\Gamma)} \quad
\end{gathered}
$$


We next demonstrate (82) (and hence (10)). It suffices to consider $y \in$ $\left[y_{N, i}, y_{N, i+1}\right]$ for $0 \leq i \leq N-1$. On $\left[y_{N, i}, y_{N, i+1}\right]$ only $\left|\chi_{N, i}^{\prime}(y)\right|$ and $\left|\chi_{N, i+1}^{\prime}(y)\right|$ contribute to the sum; furthermore we have $\left|\chi_{N, i}^{\prime}(y)\right|=\left|\chi_{N, i+1}^{\prime}(y)\right|=1 / h=$ $N / 2$, from where the result (82) follows.

\section{A.2 Piecewise quadratic interpolation}

We consider piecewise quadratic interpolation over equidistant interpolation nodes $y_{N, i}=(2 i / N-1) \in \Gamma, 0 \leq i \leq N$. We consider $N$ equal such that we may divide $\Gamma$ into $N / 2$ intervals $\left[y_{N, i}, y_{N, i+2}\right]$, for $i=0,2,4, \ldots, N-2$. The characteristic functions $\chi_{N, i}$ are for $y \in\left[y_{N, i}, y_{N, i+2}\right]$ given as

$$
\begin{aligned}
\chi_{N, i}(y) & =\frac{\left(y-y_{N, i+1}\right)\left(y-y_{N, i+2}\right)}{2 h^{2}}, \\
\chi_{N, i+1}(y) & =\frac{\left(y-y_{N, i}\right)\left(y-y_{N, i+2}\right)}{-h^{2}}, \\
\chi_{N, i+2}(y) & =\frac{\left(y-y_{N, i}\right)\left(y-y_{N, i+1}\right)}{2 h^{2}},
\end{aligned}
$$

for $i=0,2,4, \ldots, N$, where $h=2 / N=y_{N, j+1}-y_{N, j}, 0 \leq j \leq N-1$.

We recall the results (16) and (17) from Section 2.2. Let $f: \Gamma \rightarrow \mathbb{R}$ with $f \in C^{2}(\Gamma)$ and assume that $\sup _{y \in \Gamma}\left|f^{\prime \prime \prime}(y)\right|<\infty$. We then have, for any $y \in \Gamma$ and any $N \geq 0$,

$$
\left|f^{\prime}(y)-\left(I_{N} f\right)^{\prime}(y)\right| \leq 28 \frac{\left\|f^{\prime \prime \prime}\right\|_{L^{\infty}(\Gamma)}}{N^{2}} .
$$

Further, for all $y \in \Gamma$, the characteristic functions $\chi_{N, i}, 0 \leq i \leq N$, satisfy

$$
\sum_{i=0}^{N}\left|\chi_{N, i}^{\prime}(y)\right|=\frac{5}{2} N .
$$

We first demonstrate (90). It suffices to consider the interpolant $I_{N} f(y)$ for $y \in \Gamma_{i} \equiv\left[y_{N, i}, y_{N, i+2}\right]$, in which case

$$
I_{N} f(y)=f\left(y_{N, i}\right) \chi_{N, i}(y)+f\left(y_{N, i+1}\right) \chi_{N, i+1}(y)+f\left(y_{N, i+2}\right) \chi_{N, i+2}(y) .
$$

Insertion of (87)-(89) and differentiation yields

$$
\begin{aligned}
& \left(I_{N} f\right)^{\prime}(y)=\frac{1}{2 h^{2}}\left(f\left(y_{N, i}\right)\left(2 y-y_{N, i+1}-y_{N, i+2}\right)\right. \\
& \left.\quad-2 f\left(y_{N, i+1}\right)\left(2 y-y_{N, i}-y_{N, i+2}\right)+f\left(y_{N, i+2}\right)\left(2 y-y_{N, i}-y_{N, i+1}\right)\right) .
\end{aligned}
$$


We next write $f\left(y_{N, i}\right), f\left(y_{N, i+1}\right)$, and $f\left(y_{N, i+2}\right)$ as Taylor series around $y$ as

$$
\begin{aligned}
f\left(y_{N, i}\right) & =\sum_{j=0}^{2} \frac{f^{(j)}(y)}{j !}\left(y_{N, i}-y\right)^{j}+\int_{y}^{y_{N, i}} f^{\prime \prime \prime}(t) \frac{\left(y_{N, i}-t\right)^{2}}{2} d t, \\
f\left(y_{N, i+1}\right) & =\sum_{j=0}^{2} \frac{f^{(j)}(y)}{j !}\left(y_{N, i+1}-y\right)^{j}+\int_{y}^{y_{N, i+1}} f^{\prime \prime \prime}(t) \frac{\left(y_{N, i+1}-t\right)^{2}}{2} d t, \\
f\left(y_{N, i+2}\right) & =\sum_{j=0}^{2} \frac{f^{(j)}(y)}{j !}\left(y_{N, i+2}-y\right)^{j}+\int_{y}^{y_{N, i+2}} f^{\prime \prime \prime}(t) \frac{\left(y_{N, i+2}-t\right)^{2}}{2} d t .
\end{aligned}
$$

We may then insert the expressions (94)-(96) into (93) to obtain

$$
\begin{gathered}
\left(I_{N} f\right)^{\prime}(y)-f^{\prime}(y)=\frac{1}{2 h^{2}}\left(\left(2 y-y_{N, i+1}-y_{N, i+2}\right) \int_{y}^{y_{N, i}} f^{\prime \prime \prime}(t) \frac{\left(y_{N, i}-t\right)^{2}}{2} d t\right. \\
-2\left(2 y-y_{N, i}-y_{N, i+2}\right) \int_{y}^{y_{N, i+1}} f^{\prime \prime \prime}(t) \frac{\left(y_{N, i+1}-t\right)^{2}}{2} d t \\
\left.+\left(2 y-y_{N, i}-y_{N, i+1}\right) \int_{y}^{y_{N, i+2}} f^{\prime \prime \prime}(t) \frac{\left(y_{N, i+2}-t\right)^{2}}{2} d t\right) .
\end{gathered}
$$

(For $j=0$ and $j=2$ the terms on the right-hand-side of (93) cancel, and for $j=1$ we obtain $f^{\prime}(y)$.) We further bound (97) as

$$
\begin{gathered}
\left|\left(I_{N} f\right)^{\prime}(y)-f^{\prime}(y)\right| \leq \frac{\left\|f^{\prime \prime \prime}\right\|_{L^{\infty}(\Gamma)}}{4 h^{2}} \max _{y \in \Gamma_{i}}\left(\left|2 y-y_{N, i+1}-y_{N, i+2}\right|\left|y_{N, i}-y\right|^{3}\right. \\
\left.+2\left|2 y-y_{N, i}-y_{N, i+2}\right|\left|y_{N, i+1}-y\right|^{3}+\left|2 y-y_{N, i}-y_{N, i+1}\right|\left|y_{N, i+2}-y\right|^{3}\right) \\
\leq \frac{\left\|f^{\prime \prime \prime}\right\|_{L^{\infty}(\Gamma)}}{4 h} \max _{y \in \Gamma_{i}}\left(3\left|y_{N, i}-y\right|^{3}+4\left|y_{N, i+1}-y\right|^{3}+3\left|y_{N, i+2}-y\right|^{3}\right) \\
\leq \frac{\left\|f^{\prime \prime \prime}\right\|_{L^{\infty}(\Gamma)}}{4 h}\left(3(2 h)^{3}+4 h^{3}\right)=28 \frac{\left\|f^{\prime \prime \prime}\right\|_{L^{\infty}(\Gamma)}}{N^{2}},
\end{gathered}
$$

which is the desired result.

We next demonstrate (91). It again suffices to consider $y \in \Gamma_{i}$. On $\Gamma_{i}$ only $\chi_{N, i}^{\prime}(y), \chi_{N, i+1}^{\prime}(y)$, and $\chi_{N, i+2}^{\prime}(y)$ contribute to the sum. With $h=2 / N=$ $y_{j+1}-y_{j}, 0 \leq j \leq N-1$, we have

$$
\begin{gathered}
\max _{y \in \Gamma_{i}}\left|\chi_{N, i}^{\prime}(y)\right|=\frac{N^{2}}{8} \max _{y \in \Gamma_{i}}\left|2 y-y_{N, i+1}-y_{N, i+2}\right|=\frac{3}{4} N, \\
\max _{y \in \Gamma_{i}}\left|\chi_{N, i+1}^{\prime}(y)\right|=\frac{N^{2}}{4} \max _{y \in \Gamma_{i}}\left|2 y-y_{N, i}-y_{N, i+2}\right|=N, \\
\max _{y \in \Gamma_{i}}\left|\chi_{N, i+2}^{\prime}(y)\right|=\frac{N^{2}}{8} \max _{y \in \Gamma_{i}}\left|2 y-y_{N, i}-y_{N, i+1}\right|=\frac{3}{4} N .
\end{gathered}
$$

The result then follows. 


\section{A.3 Proof that $\mathcal{W}(\xi)<\log (\xi)$ for real $\xi>e$}

We recall the definition of the LambertW function

$$
\xi=\mathcal{W}(\xi) e^{\mathcal{W}(\xi)}, \quad \xi \in \mathbb{C} .
$$

By implicit differentiation we obtain

$$
\mathcal{W}^{\prime}(\xi)=\frac{1}{e^{\mathcal{W}(\xi)}+\xi}
$$

for $\xi \neq-1 / e$. Further, $\mathcal{W}(\xi)$ is real-valued for real-valued $\xi>0$. Hence

$$
\mathcal{W}^{\prime}(\xi)<\frac{1}{\xi}
$$

for real $\xi>0$. We then make the observation that

$$
\mathcal{W}(e)=\log (e)=1 \text {. }
$$

Hence for all $\xi>e$, we have $\mathcal{W}(\xi)<\log (\xi)$.

\section{References}

[1] M. Barrault, N. C. Nguyen, Y. Maday, and A. T. Patera. An "empirical interpolation" method: Application to efficient reduced-basis discretization of partial differential equations. C. R. Acad. Sci. Paris, Série I., 339:667$672,2004$.

[2] C. Bernardi and Y. Maday. Spectral methods. In Handbook of numerical analysis, Vol. V, Handb. Numer. Anal., V, pages 209-485. North-Holland, Amsterdam, 1997.

[3] J. Borggaard and J. Burns. A PDE sensitivity equation method for optimal aerodynamic design. J. Comput. Phys., 136(2):366-384, 1997.

[4] C. Canuto, M. Y. Hussaini, A. Quarteroni, and T. A. Zang. Spectral methods. Scientific Computation. Springer-Verlag, Berlin, 2006. Fundamentals in single domains.

[5] R. Corless, G. Gonnet, D. Hare, D. Jeffrey, and D. Knuth. On the Lambert $W$ function. Advances in Computational Mathematics, 5:329-359, 1996.

[6] J. L. Eftang, M. A. Grepl, and A. T. Patera. A posteriori error bounds for the empirical interpolation method. Comptes Rendus Mathematique, 348(9-10):575-579, 2010.

[7] M. Grepl. A Posteriori Error Bounds for Reduced-Basis Approximations of Nonaffine and Nonlinear Parabolic Partial Differential Equations. MЗAS, Submitted, 2010. 
[8] M. A. Grepl, Y. Maday, N. C. Nguyen, and A. T. Patera. Efficient reduced basis treatment of nonaffine and nonlinear partial differential equations. ESAIM: M2AN, 41(3):575-605, 2007.

[9] Y. Maday, N. C. Nguyen, A. T. Patera, and G. S. H. Pau. A general multipurpose interpolation procedure: The magic points. Communications in pure and applied mathematics, 8:383-404, 2009.

[10] A. Quarteroni, R. Sacco, and F. Saleri. Numerical Mathematics, volume 37 of Texts Appl. Math. Springer, New York, 1991.

[11] S. C. Reddy and J. A. C. Weideman. The accuracy of the Chebyshev differencing method for analytic functions. SIAM J. Numer. Anal., 42(5):21762187 (electronic), 2005.

[12] T. J. Rivlin. The Chebyshev polynomials. Wiley-Interscience [John Wiley \& Sons], New York, 1974. Pure and Applied Mathematics.

[13] G. Rozza, D. B. P. Huynh, and A. T. Patera. Reduced Basis Approximation and a posteriori Error Estimation for Affinely Parametrized Elliptic Coercive Partial Differential Equations. Archives of Computational Methods in Engineering, 15(3):229-275, 2008.

[14] E. Tadmor. The exponential accuracy of Fourier and Chebyshev differencing methods. SIAM J. Numer. Anal., 23(1):1-10, 1986. 